\title{
Ionospheric tomography and its applications in radio science and geophysical investigations
}

\author{
Leonard Kersley \\ Radio and Space Physics Group, Department of Physics, University of Wales, Aberystwyth, U.K.
}

\begin{abstract}
The paper reviews recent progress in the development of radio tomographic imaging of the ionized atmosphere, particularly in relation to the use of the technique to assist in the mitigation of propagation effects on practical radio systems.
\end{abstract}

Key words ionospheric tomography - total electron content - ionospheric propagation - ionospheric modeling

\section{Introduction}

The development of tomographic imaging has been one of the great scientific achievements of the final decades of the twentieth century. While the mathematical basis of the method was formulated early in the century, the advances in computing technology in the 1960s gave practical realization to the technique as an imaging tool. The method is hailed primarily for transforming medical diagnostics, but it has also lead to significant progress in many other fields, including geophysics. The underlying principles behind the method and descriptions of some of the early applications can be found in the special issue of Proceeding of the IEEE, 71 (3), 1983. Further material of a fundamental

Mailing address: Prof. Leonard Kersley, Radio and Space Physics Group, Department of Physics, University of Wales, Aberystwyth, Dyfed SY23 3BZ, U.K.; e-mail: lek@aber.ac.uk nature can also be found in the book by Kak and Slaney (1988).

A number of authors have reviewed the application of the method to radio tomographic imaging of the ionized atmosphere. Early developments in the field are documented in special issues of the journals International Journal of Imaging Systems and Technology, 5 (2), 1994 and Annales Geophysicae, 13 (12), 1996, while in further reviews Leitinger $(1996,1999)$ discussed the theoretical basis and the limitations of the ionospheric application. More recently, ionospheric tomography, including comprehensive descriptions of a number of theoretical formulations, has been the subject of a book by Kunitsyn and Tereshchenko (2003). In addition, Pryse (2003) has reviewed experimental results from the application of radio tomography to ionospheric imaging, concentrating in the main on use of the method in investigations directed towards understanding of the underlying geophysics. The present paper aims to focus on aspects of the use of tomographic imaging as an aid to practical radio science applications. Material relating to purely geophysical studies of the ionized atmosphere, which was presented in the oral version of the paper, can be found in the earlier review by Pryse (2003) and will not be repeated here. 
The experimental results discussed here involve measurements of the line integral of the electron density made along ray paths to ground stations from satellites in low Earth polar orbits. The Total Electron Content (TEC) is determined using the phase coherent signals from satellites in the Navy Ionospheric Monitoring System (NIMS), monitored at a chain of receiving locations aligned essentially in longitude, but separated in latitude. Inversion of the data set in a reconstruction algorithm yields a pixelised image in two dimensions of the spatial distribution of the electron density throughout the region of the ray-path intersections. While many groups worldwide have been involved in the development of radio tomographic imaging and the technique has now been used in many situations, the results presented here are concerned in the main with investigations of the potential usefulness of the method to applications related to practical radio systems. The experimental observations have been made for the most part at locations instrumented by the UWA group in U.K. and Scandinavia. Maps with the current stations operated by the group are shown in fig. 1 , though it should be noted that some of the work discussed here relates to results from earlier measurements, including those made at five locations in U.K. spanning the latitudinal range between about $50^{\circ} \mathrm{N}$ and $61^{\circ} \mathrm{N}$ and campaigns involving additional sites in mainland Sweden.

The development work carried out in recent years has demonstrated that radio tomography has now matured into a powerful experimental technique capable of providing images of largescale spatial structures in the ionosphere from a limited number of ground stations. The spatial nature of the observations is complementary to those from many other types of experimental measurements of the ionosphere. The limitations to the method were thoroughly investigated and well understood from early simulation studies. These result primarily from the limited-angle geometry of the satellite-to-ground observations so that there is incomplete information in the raw measurements about the vertical profile. However, even at an early stage of the development, algorithms were formulated capable of re-
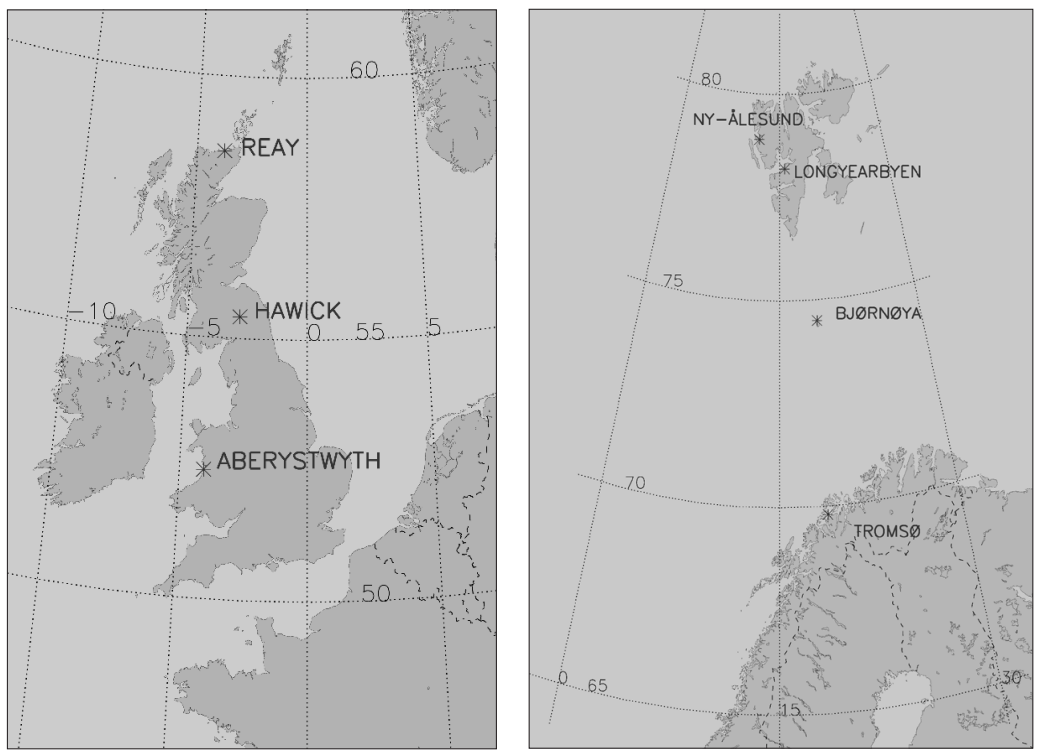

Fig. 1. Maps showing locations of satellite receiving station chains in U.K. and Scandinavia currently in use by UWA group. 
producing ionospheric layers at different heights throughout the image (Fremouw et al., 1993). In addition, the temporal coverage is dependent on the number and orbits of the available satellites with appropriate beacon transmitters.

\section{Tomography and ionosondes}

The ionosonde remains the basic experimental tool used to monitor the state of the ionized atmosphere for radio systems applications.
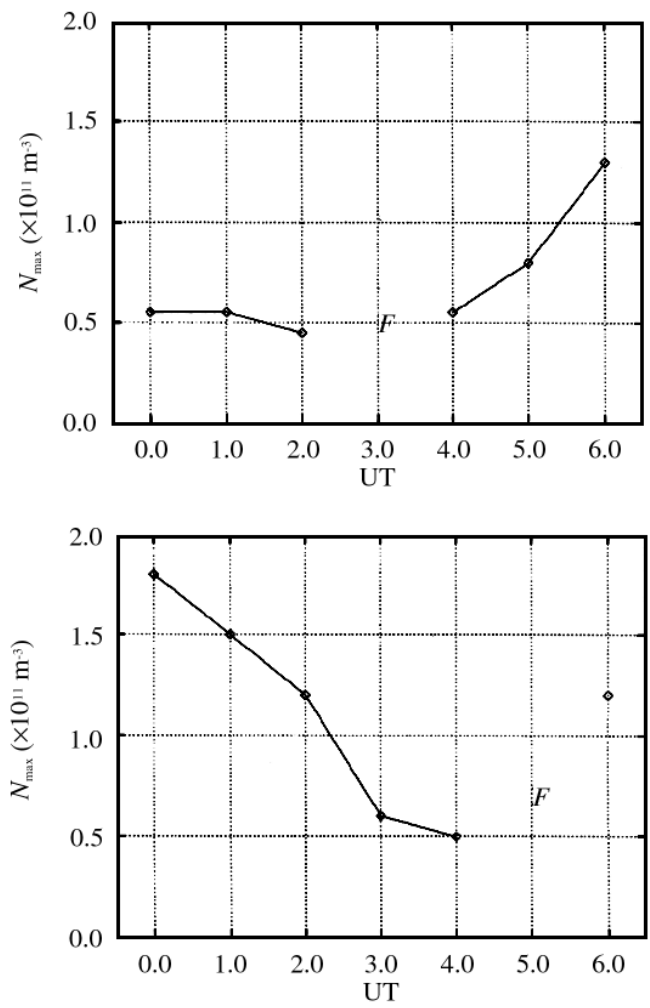

Fig. 2. Estimates of peak electron density $(N m F 2)$ obtained from the ionosondes at Chilton (left upper) and Lannion (left lower) as a function of time, together with (right) tomographic images from satellite passes at three times throughout the night. The $F$ denotes spread- $F$ conditions. From Kersley et al. (1997), reproduced by permission of the American Geophysical Union.
Significant advances have been made in recent years in the development of the instrument, not just in the electronic hardware and digital processing of the sounding signals, but also in inversion software that can yield electron density versus height profiles below the layer peak in near-real time. However, ionosondes can only provide measurements for specific locations at discrete times. The spatial imaging capability of the radio tomography technique forms a natural complement to the ionosonde for routine monitoring, with synergies of mutual benefit to

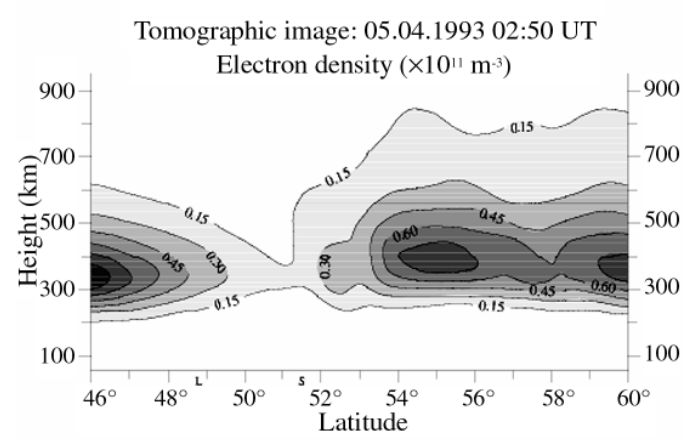

Tomographic image: 05.04.1993 03:20 UT

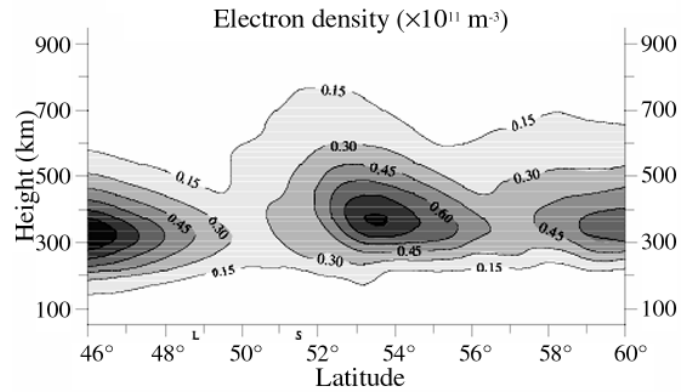

Tomographic image: 05.04.1993 04:39 UT Electron density $\left(\times 10^{11} \mathrm{~m}^{-3}\right)$

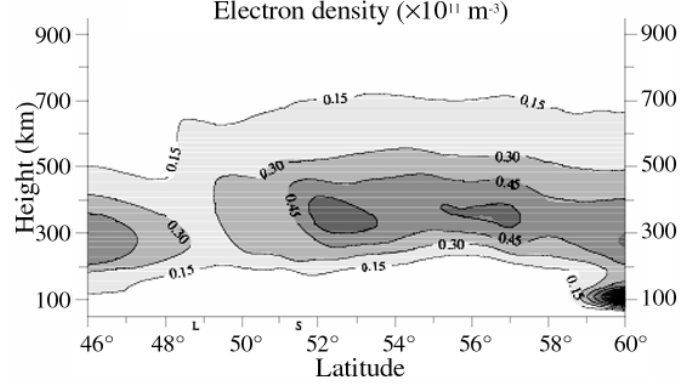


both methods. The addition of information about the horizontal structure of the ionosphere from tomographic imaging can be used to place the point measurements from the sounder in their wider spatial context, while inclusion of input from an ionosonde in the reconstruction algorithm can be used to mitigate the limited capability of the tomographic technique to determine heights.

The complementary nature of the two experimental techniques is demonstrated in fig. 2 . The upper and lower panels to the left show respectively measurements of the electron density at the layer peak $(N m F 2)$ obtained from the ionosondes at Chilton $\left(51.5^{\circ} \mathrm{N}\right)$ from Lannion $\left(48.8^{\circ} \mathrm{N}\right)$, as a function of time during a night of very severe geomagnetic disturbance. The interpretation of these observations can be elucidated from the tomographic images obtained from satellite passes at three times throughout the night (Kersley et al., 1997). The spatial images show that the spread- $F$ conditions are related to irregularities at the poleward edge of the trough, while it is a boundary blob that is responsible for the increased ionisation seen later in the night at Slough, during these exception conditions where the trough minimum was found over Northern France.

Measurements by ionosondes have also been used to improve the representation of the vertical profile in tomographic reconstructions. Discussion of the role of ionosonde input in reconstruction algorithms, used as complementary information to the TEC observations, can be found in Kersley et al. (1993). The paper was also the first of several that include verification of the resultant image of electron density using independent results from the EISCAT incoherent scatter radar.

\section{Tomography and empirical or parameterised models}

Radio tomography is an experimental technique that can be used to provide independent measurements with which to test the functionality and reliability of the empirical and parameterized models that are in use for the mitigation of ionospheric effects on radio systems.
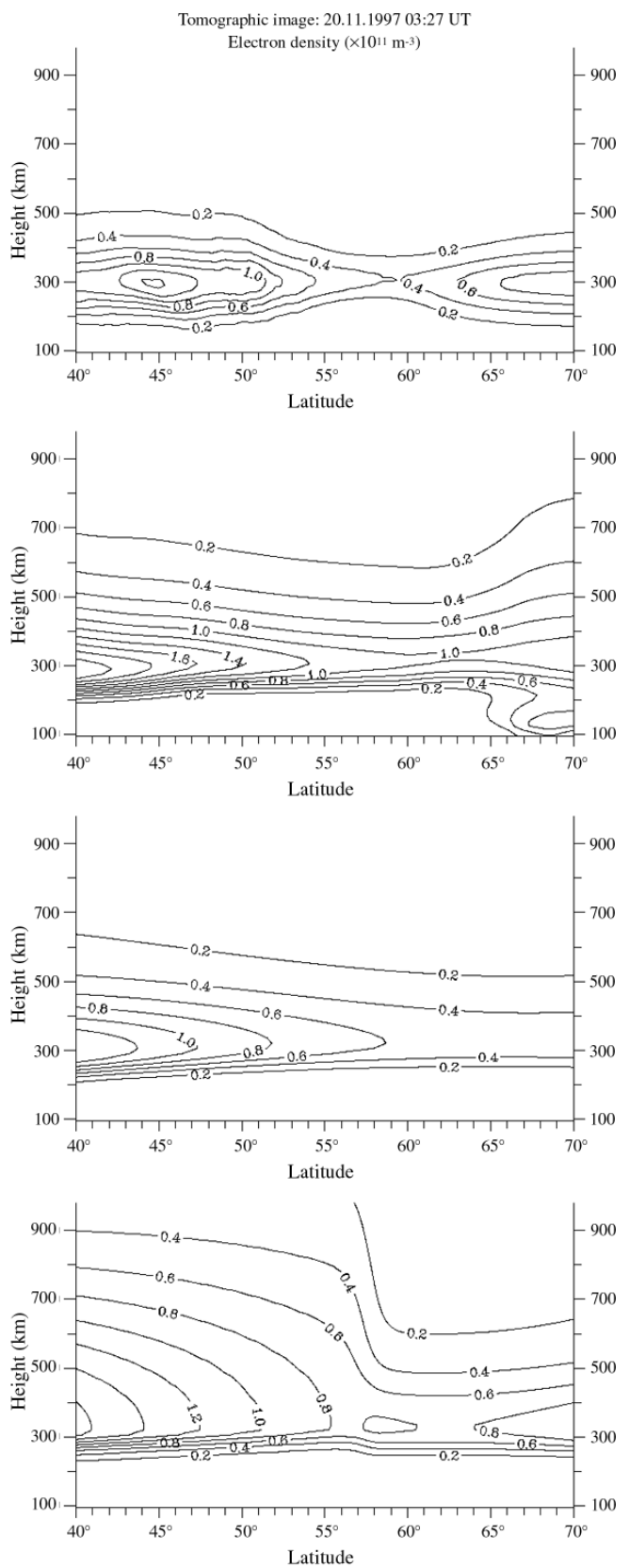

Fig. 3. An actual tomographic image of electron densities (upper panel), with corresponding outputs from three ionospheric models: (in descending order of panels) IRI-95, PIM and COST 238. From Dabas and Kersley (2003), reproduced by permission of the American Geophysical Union. 
The region of the main trough, a dynamic feature whose location and structure is highly variable and which is found over Northern Europe on many nights, presents particular problems for such models. Most models do not even attempt to incorporate the feature, while for others, placing the trough in the wrong place or with inappropriate gradients may represent a poorer solution for a particular radio application than complete omission of the feature. Tomographic imaging of large-scale spatial structures like the main trough provides a powerful tool to aid the verification of such models by comparison with actual observations.

The panels of fig. 3, taken from Dabas and Kersley (2003), show an example of such comparison between a tomographic image and three different ionospheric models, the International Reference Ionosphere (IRI-95) (Bilitza et al., 1993), the Parameterised Ionospheric Model (PIM) (Daniell et al., 1995) and the European regional model developed during the COST 238 Action (Bradley, 1999). The experimental results from observation at a tomographic chain of stations in U.K. show an ionosphere with a clear trough and in addition a mid-latitude nighttime enhancement to the south. The output from the IRI-95 model for the same geophysical conditions fails to replicate either feature, while that from PIM does show a trough, but with no localized enhancement to the south. The results from the COST 238 model do appear to show a trough, though extreme caution must be used in the interpretation because this particular model was designed specifically to cover the European region below $55^{\circ} \mathrm{N}$ latitude, with default matching to the IRI model above that latitude, so that the feature shown is likely to be an artifact of this process.

\section{Tomography and the mapping of ionospheric parameters}

Maps of ionospheric parameters, on a regional or global basis, provide another tool that is used to correct for propagation effects in practical radio systems. Dabas and Kersley (2003) also investigated the potential use of tomographic images in mapping. They demonstrated that improvements could be achieved to the reliability of maps of the F2-layer critical frequency and peak electron density over Europe by incorporating information from experimental tomography into the mapping process. Figure 4 shows a comparison of a map of $N m F 2$, predicted by the IRI-95 model for particular conditions, with one in which an actual
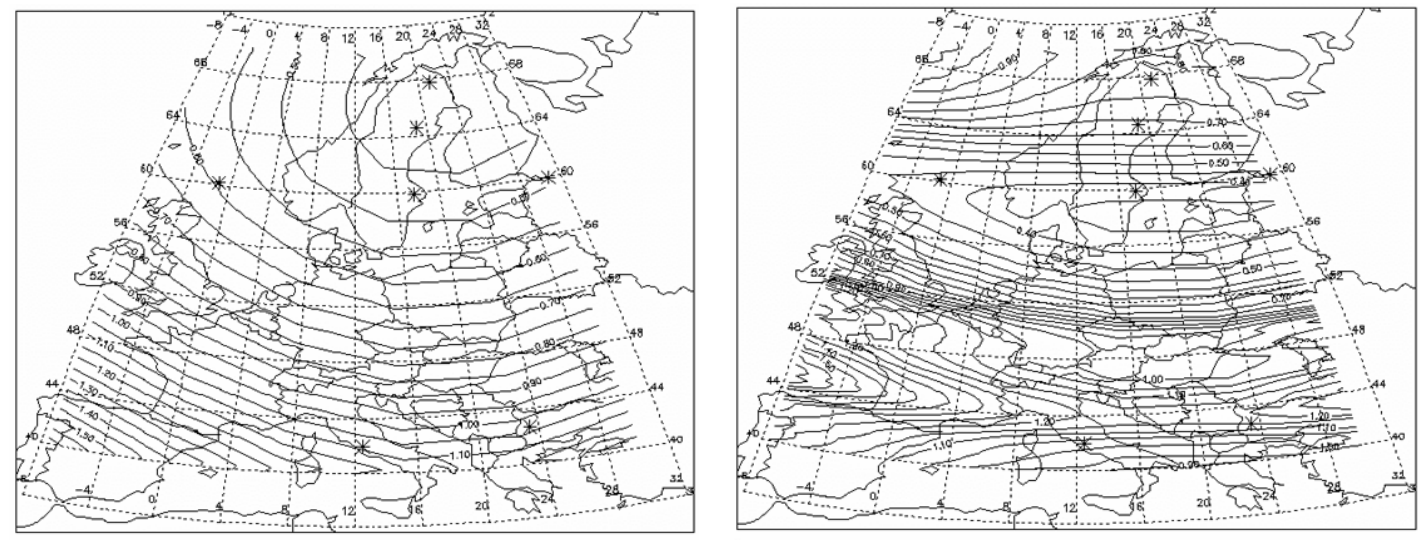

Fig. 4. Contour maps of $N m F 2$ over Europe obtained from IRI-95 alone (left) and using input from an actual tomographic image plus the IRI-95 model (right). From Dabas and Kersley (2003), reproduced by permission of the American Geophysical Union. 

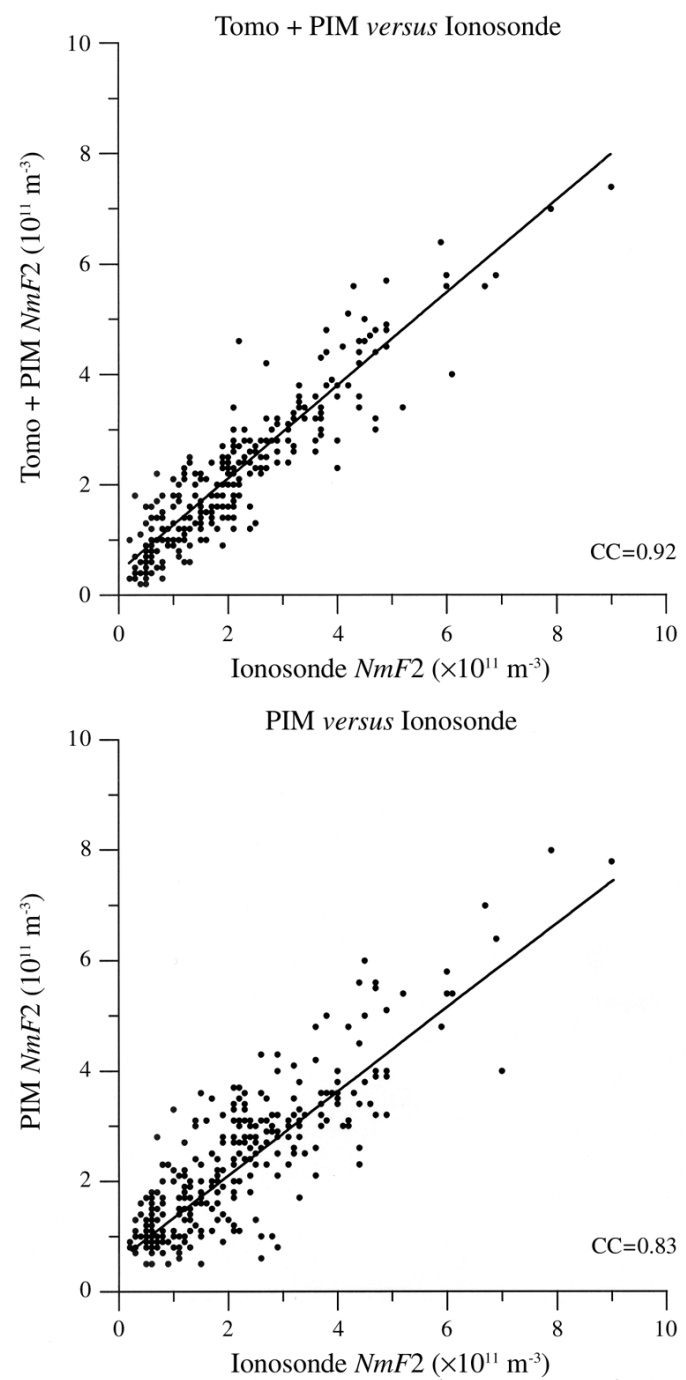

Fig. 5. Correlation of $N m F 2$ from tomography plus PIM maps (upper) and PIM alone maps (lower) with corresponding values from ionosondes. From Dabas and Kersley (2003), reproduced by permission of the American Geophysical Union

tomographic image obtained from observations made in U.K. has been used to provide the latitudinal gradient. In essence, the electron densities at the layer peak, determined from the image, were mapped in longitude, with zonal gra- dients from the model being used to create a revised map that was more realistic of the actual conditions. The locations of the spatial structures that were not present in the model output alone can be identified from the map modified by the use of tomographic input.

Verification of the improvement afforded by the use of tomographic observations in the mapping procedure was obtained by comparing the mapped values with actual measurements of $f_{o} F 2$ from the network of ionosondes throughout $\mathrm{Eu}-$ rope. Figure 5 shows the results of such comparison for the PIM model. It can be seen that inclusion of tomographic input with the PIM model improved the correlation coefficient between the mapped $N m F 2$ and actual ionosonde measurements to 0.92 , while a smaller coefficient of 0.83 was found when the PIM model was used alone.

\section{Tomography and oblique ionograms}

The possible role of radio tomography as an aid to oblique ionospheric sounding has been investigated by Heaton et al. (2001). A network of IRIS oblique sounders was established in U.K.

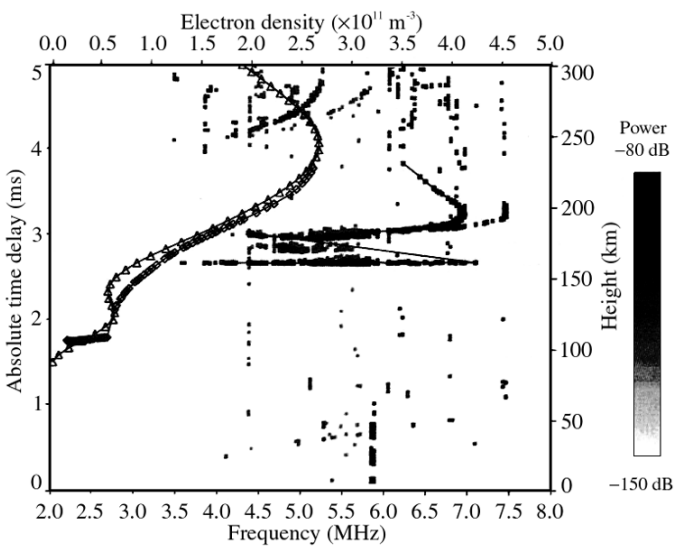

Fig. 6. An oblique ionogram and bottomside profile of electron density from IRIS observations in U.K., together with a complete profile at the midpoint obtained from a corresponding tomographic image. From Heaton et al. (2001), reproduced by permission of the American Geophysical Union. 


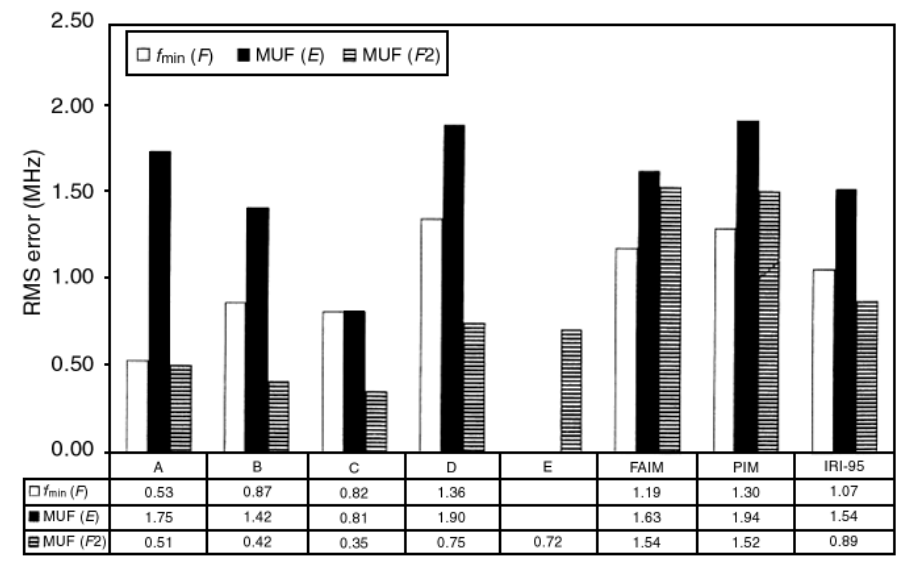

Fig. 7. Histograms of RMS errors in the estimation of the maximum useable frequency showing that use of tomographic images gives smaller errors for F2-layer MUF than the FAIM, PIM and IRI 95 models. From Rogers et al. (2001), reproduced by permission of the American Geophysical Union.

during an experimental campaign, covering the region spanned by a chain of tomographic receivers. The results demonstrated that the maximum density at the mid-point of the oblique path estimated by inversion of the oblique ionogram was in reasonable agreement with the density at the corresponding height and latitude in the tomographic image. The example shown in fig. 6 confirms this finding, though discrepancies can be seen at $E$-layer heights. It was concluded from the study that images of path conditions from the tomographic technique could have a role to play in assessing the applicability of the assumption of spherical symmetry in the reduction of oblique incidence ionograms.

\section{Tomography and $H F$ ray tracing}

The observations from the IRIS oblique incidence sounder network in U.K. were also used in a study of the potential of radio tomography in aiding the ray tracing of $H F$ propagation paths. The results are discussed in detail in Rogers et al. (2001). The study investigated the effects of various types of input information that can be used to constrain the vertical electron density structure in the tomographic reconstruc- tions. It was found that the use of a fine height resolution $(5 \mathrm{~km})$ and incorporation of input from one vertical ionosonde in the reconstruction process made significant improvements to the overall reliability of the tomographic image. As expected, $E$-layer propagation was better defined using a climatological model than by the tomographic method. It can be seen from fig. 7 that the use of tomographic images reduced the RMS error in the determination of the F2-layer Maximum Useable Frequency (MUF), yielding significantly smaller errors than those obtained from three independent ionospheric models (FAIM, PIM and IRI-95).

\section{Tomography and $H F$ direction finding}

Warrington et al. (2002) presented observations from an $H F$ direction-finding experiment, on a link between Uppsala in Sweden and Leicester in U.K., where the propagation path was aligned approximately with the orientation of the main ionospheric trough. An example is shown here that demonstrates significant deviations from the great-circle path. The results from the days 326-327, reproduced here in fig. 8 , show anomalous out-of-great-circle propaga- 

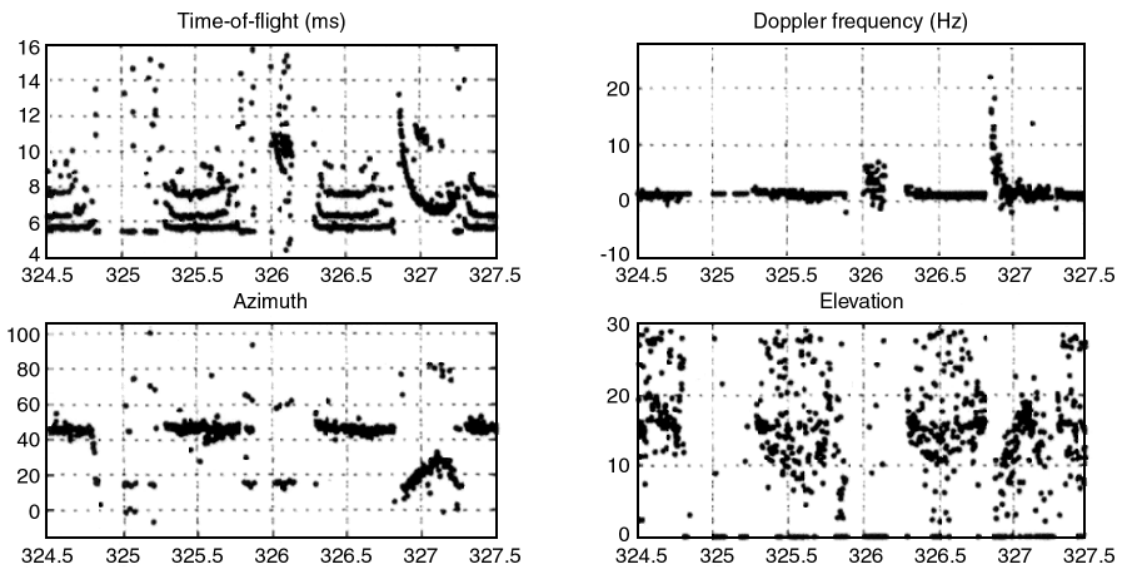

Fig. 8. Results from an $H F$ direction-finding experiment between Uppsala to Leicester during a three-day period in November 2001. Note in particular the anomalous azimuthal directions of the received signals during the night of 326-327. From Warrington et al. (2002), reproduced by permission of the authors.
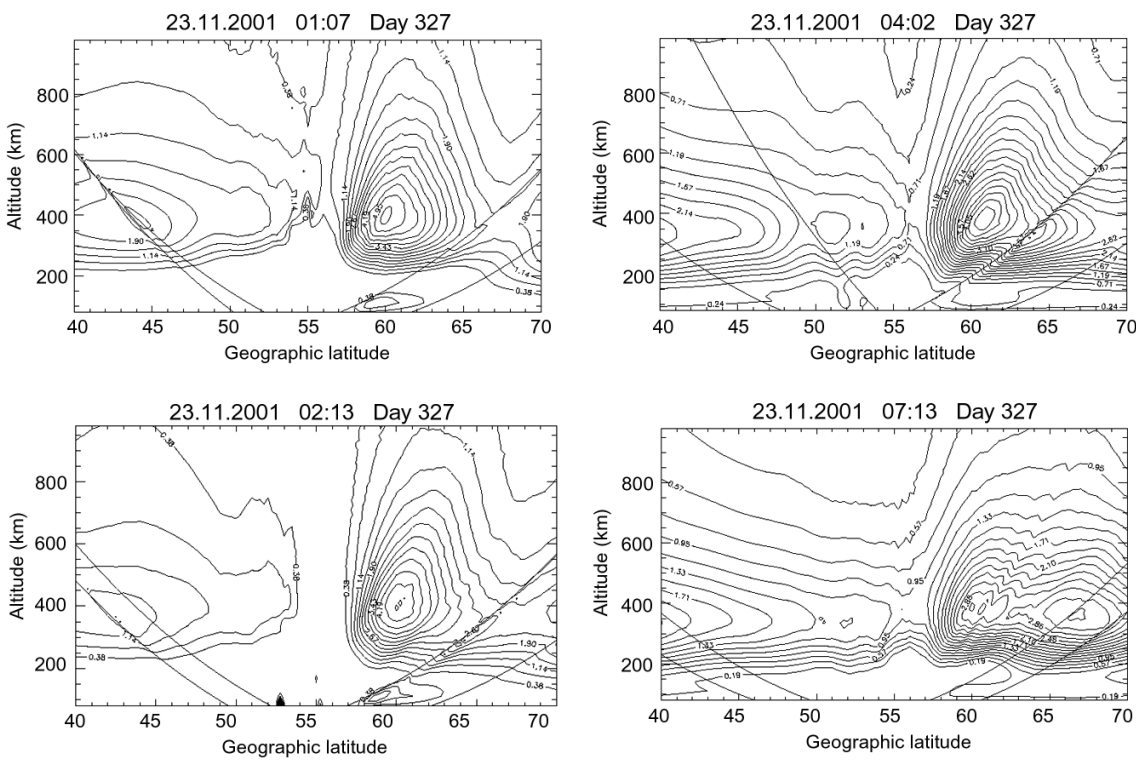

Fig. 9. A sequence of tomographic images from four satellite passes throughout the night of 326-327, monitored at the stations in U.K., which show a deep trough with a strong ionisation enhancement or boundary blob polewards of the minimum that is acting as a reflector for the out-of-great-circle $H F$ propagation found on the Uppsala to Leicester path shown in fig. 8.

tion during the night, with consequent increased times of flight and doppler frequency shifts. The azimuth of the received signals shows a sudden transition from the great-circle direction, followed by a smooth rotation and reversal over a period of several hours before returning to the original direction. In a subsequent study, images of electron density from 

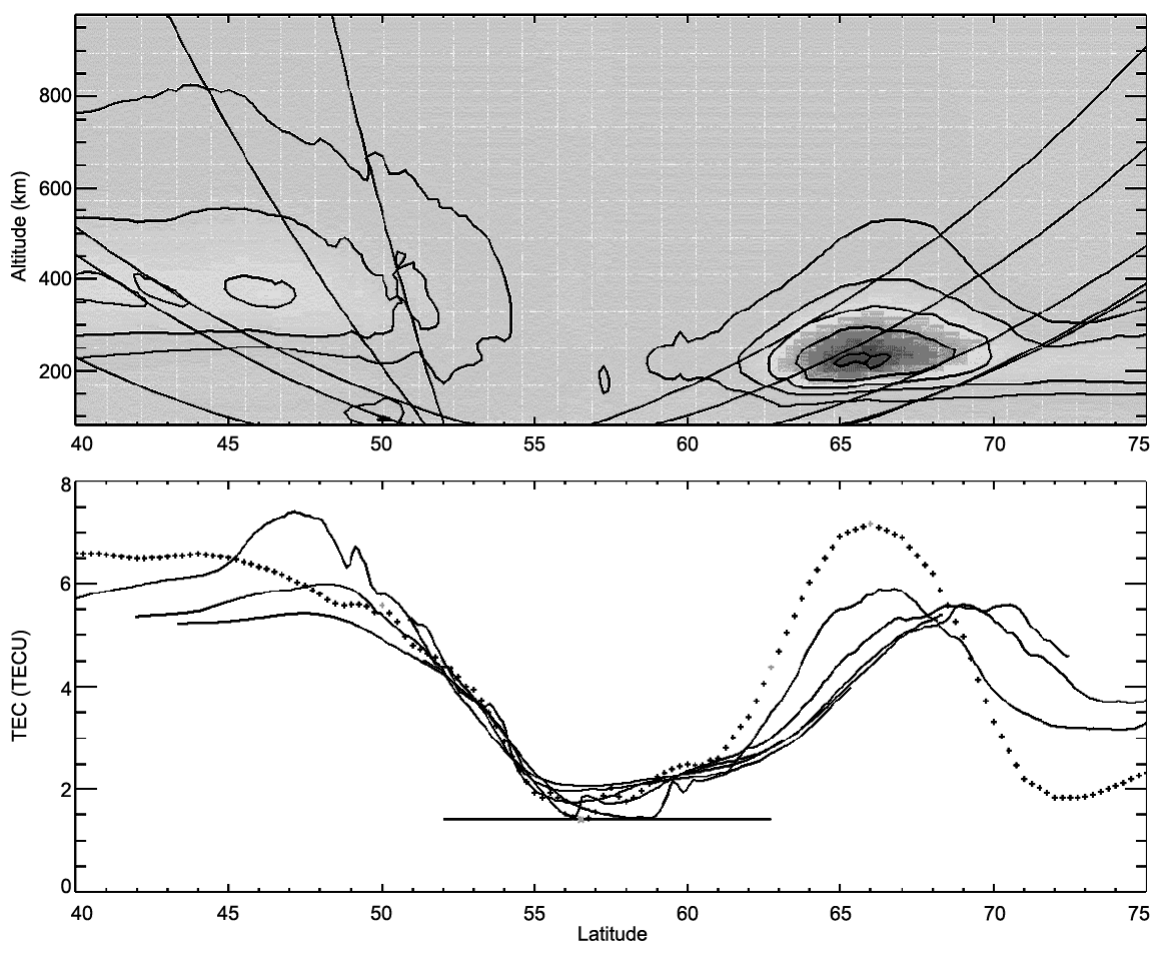

Fig. 10. Tomographic image from observations in U.K. (upper panel), with plots of equivalent vertical total electron content from the individual station observations (solid lines) and the vertical total electron content computed through the tomographic image (dotted line) shown in the lower panel. From Kersley et al. (2002).

the U.K. tomography chain, from a succession of satellite passes throughout the night, revealed a trough with a steep poleward wall that advanced equatorwards during the early part of the night before receding again northwards (fig. 9). The anomalous propagation found in the results from the $H F$ experiment can be explained in terms of reflection by the enhanced densities to be seen in the tomographic images polewards of the trough minimum.

\section{Tomography and vertical TEC}

Kersley et al. (2002) investigated the limitations to the accuracy of experimental measurements of total electron content imposed by the necessary assumptions required to convert the slant observations to equivalent vertical measurements. The assumed height of the thin-shell ionospheric layer was found to be a critical factor. However, it was demonstrated that some advantage could be gained by assessing the vertical TEC by integration through a tomographic image as opposed to using the equivalent vertical estimate of the parameter for an assumed thin-layer height. In the example shown in fig. 10 it can be seen that to the north of the trough the tomographic image reveals that the peak height of the actual ionosphere was only some $200 \mathrm{~km}$. The lower panel shows that the resultant equivalent vertical total electron contents estimated from the slant measurements at the individual stations for an assumed ionospheric height of $350 \mathrm{~km}$ are all much less than the vertical TEC (shown by the dotted line) in that re- 
gion obtained from the tomographic image above. In a similar way, an assumed thin-shell ionosphere at too great a height leads to overestimation of the equivalent vertical TEC. It was argued that the vertical TEC obtained from the tomographic image was a more reliable estimate than the equivalent vertical TECs determined from the slant measurements.

\section{Tomography and trough parameters}

Vertical TEC estimates from tomographic images of the kind described above are being used in a project to characterize the main trough in terms of a set of defined parameters. The parameters have been chosen to identify both the location of the trough and the local structure of the ionosphere in the vicinity of the feature. In addition to the latitude of the minimum, the parameters include the gradients of both the equatorward and poleward walls and evidence for a local maximum that would represent a boundary blob poleward of the minimum. A database of the parameters has been compiled, using the observations from the tomographic chain of stations in U.K., covering more than one year. The objectives of the study are two fold. First, the aim is to use the parameters in the extensive database to try to further understanding of the behaviour of the trough under a wide variety of geophysical conditions. In addition, characterisation of the feature in this defined way is planned to provide parameters that can be calculated directly from models so that the actual observations can be used in a direct test of the reliability of the model in the trough region.

\section{Tomography and the SUCTIP model}

While the work outlined above involved the testing of models that had been developed specifically for radio systems applications, tomographic images have also been used to assess the ability of a physical model of the coupled ionosphere/thermosphere system to replicate conditions in the high-latitude ionosphere. Experimental observations from a chain of satellite stations in the Scandinavian sector were used to provide 'ground truth' information for comparison with output from the Sheffield University Coupled Thermosphere Ionosphere Plasmasphere (SUCTIP) model. Figure 11, taken from the paper by Idenden et al. (1998) reporting the results of the study, shows an example of a tomographic image containing a dayside trough in the high-latitude ionosphere that can be compared with the output from the SUCTIP model for the appropriate conditions, which is reproduced in the panel below.

A subsequent study by Balthazor et al. (2002) compared the locations of the trough minimum, determined experimentally from tomographic images made under quiet geomagnetic conditions, with those predicted by the model (fig. 12).

The results of the comparison shown in fig. 12 demonstrate that the model tends consistent-
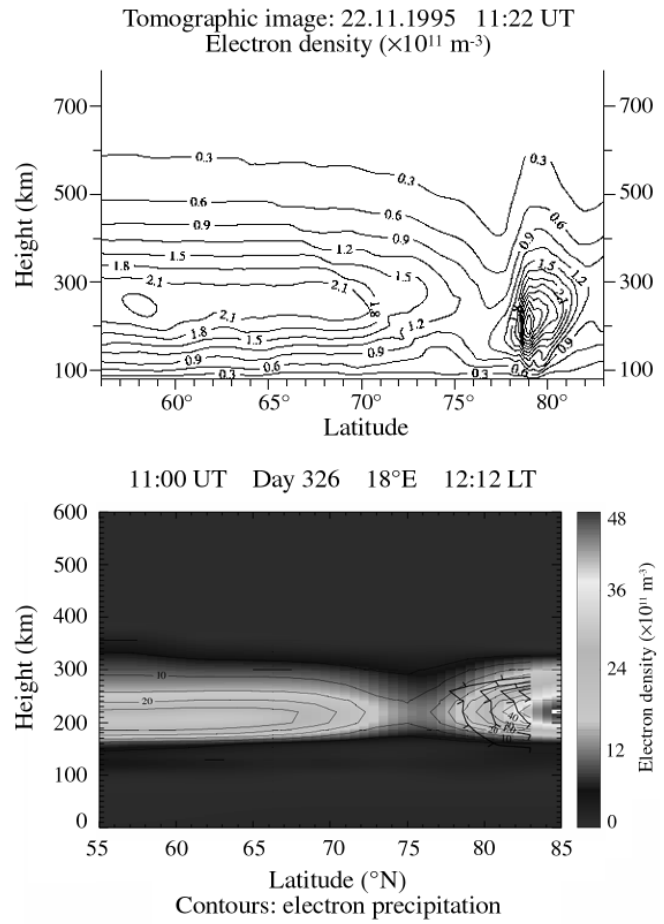

Fig. 11. Tomographic image from experimental observations in Scandinavia with corresponding output from the SUPIM model. From Idenden et al. (1998), reproduced by permission of Annales Geophysicae. 


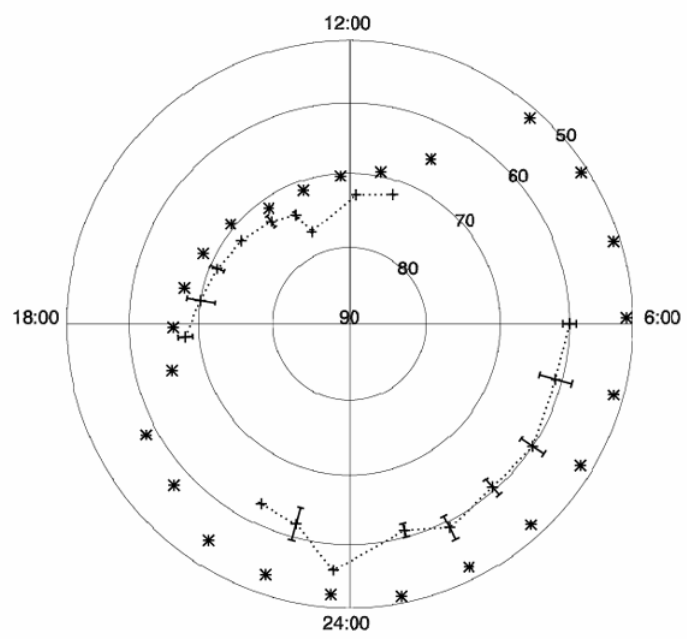

Fig. 12. Polar plot in the magnetic reference frame comparing average locations of the trough minimum under quiet geomagnetic conditions obtained from tomographic images $(+)$ with estimates by the SUCTIP model (*). From Balthazor et al. (2002).

ly to place the trough at slightly lower latitudes than those identified experimentally from the tomographic observations.

\section{Tomography and space weather}

Many of the processes that can have adverse effects on the performance of radio systems are of particular significance during times when disturbed space weather impacts on the terrestrial environment. Radio tomographic imaging of ionospheric structures found at high latitudes has been shown to provide insight into space-weather processes. The convergence of the geomagnetic field close to the Earth results in flux tubes with very different plasma populations that are characteristic of widely separate regions of space being brought into close spatial proximity in the dayside cusp ionosphere. In consequence, structures in the ionospheric plasma represent signatures of physical processes that are operating far out in space. Tomographic imaging of such spatial features has demonstrated that they contain footprints of important mechanisms in solar-ter- restrial coupling. Several papers have addressed such issues, including two that illustrate the signatures in the dayside cusp ionosphere of the reconnection processes that link the Interplanetary Magnetic Field (IMF), carried by the solar wind, to the geomagnetic field.

The location of the magnetic reconnection and the resultant behaviour and flow of the ionospheric plasma is dependent, in particular, on the orientation of the north/south- or $z$-component of the IMF. When $B z$ is directed southwards the IMF is oriented anti-parallel to the geomagnetic field at the magnetopause at low latitudes, so that the reconnection occurs close to the equatorial plane. The resultant tension exerted by the solar wind on the geomagnetic field drives the ionospheric plasma in the familiar two-cell pattern at high latitudes, with anti-sunward flow across the polar cap, as is illustrated in fig. 13.

Walker et al. (1998), using observations from the chain of tomographic stations in the vicinity of Svalbard, investigated the ionospheric footprints of low-latitude reconnection. An image, reproduced in fig. 14, shows characteristic features that can be identified with signatures of the reconnection process. The open/closed field line boundary is marked by a steep meridional gradient in the F2-layer ionospheric electron density. The increase in the peak height of the layer to the north was associated with the dispersed spectrum of the particles precipitating from the magnetopause reconnection site, with the softer energies
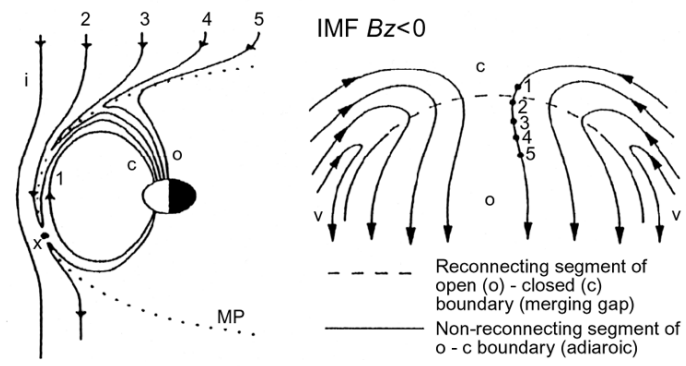

Fig. 13. Magnetic reconnection close to the equatorial plane and the consequent convection of plasma in the high-latitude ionosphere, driven in an anti-sunward flow across the polar cap. From Lockwood (1998), reproduced by permission of the publishers. 


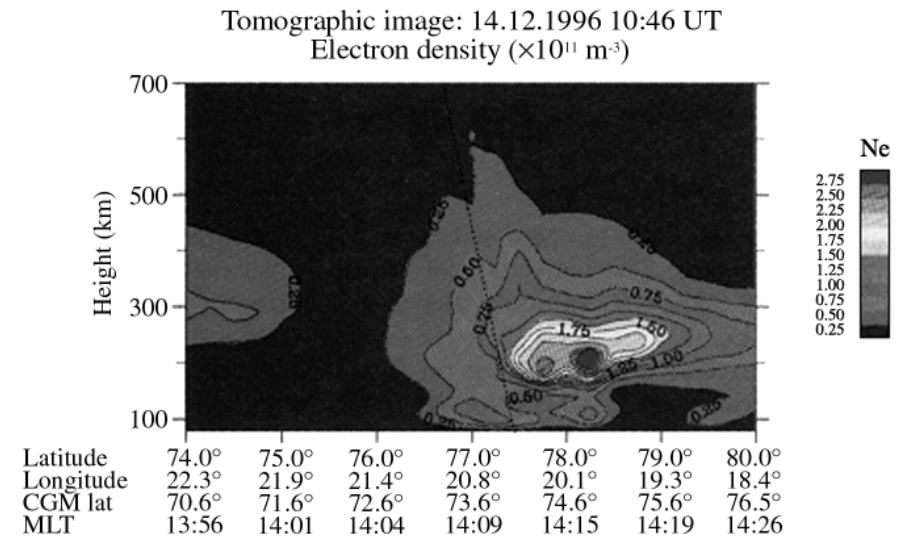

Fig. 14. Tomographic image showing signatures in the high-latitude ionosphere of reconnection at the equatorial magnetopause. From Walker et al. (1998), reproduced in modified form by permission of the American Geophysical Union.

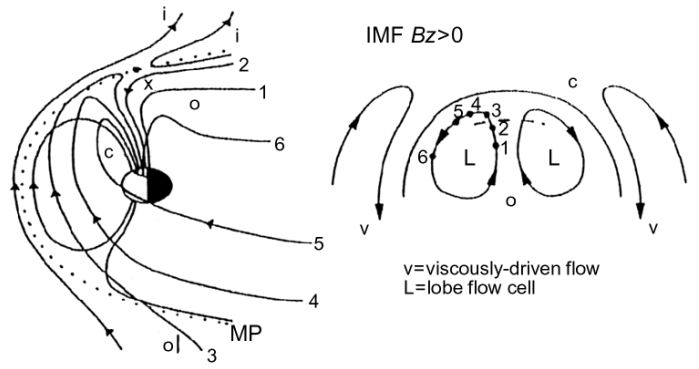

Fig. 15. Magnetic reconnection in the magnetospheric lobe and the consequent convection of plasma in the high-latitude ionosphere, which polewards of the adiaroic boundary is initially in a sunward direction. From Lockwood (1998), reproduced by permission of the publishers.

taking longer to impact the ionosphere as the flux tube is convected polewards. The tomographic image also revealed ionospheric signatures of the field-aligned current system driven by the reconnection. The $E$-layer enhancement just south of the boundary was linked to harder electron precipitation from the central plasma sheet associated with an upward current, while the narrow band of increased densities extending to high altitudes immediately polewards of the reconnection location marked a downward field-aligned current.
When the IMF is directed northwards the anti-parallel condition necessary for reconnection with the geomagnetic field is now satisfied at high latitudes in the magnetospheric lobe. The resultant lobe reconnection and the consequent convection of plasma in the polar ionosphere are illustrated in fig. 15. Pryse et al. (1999) identified a tomographic image that contained the spatial footprints in the high-latitude dayside ionosphere of lobe reconnection, which is reproduced here as fig. 16. There is a marked contrast between this signature of reconnection in the magnetospheric lobe and that shown earlier for the low-latitude situation. Figure 15 illustrates that for lobe reconnection the initial motion of the flux tubes, driven by the tension of the IMF solar wind, is equatorwards with a consequent reversed dispersion of the spectrum of the precipitation as a function of latitude. The resultant signature of this process can be seen in the tomographic image, where the increasing peak height of plasma in the flux tubes convecting from the north towards the adiaroic boundary reflects the softening of the precipitation driven by the reconnection process. The adiaroic boundary, across which there is no transfer of flux, is marked by a steep gradient in the density. Plasma from the south is driven in the anti-sunward direction up to the boundary and 


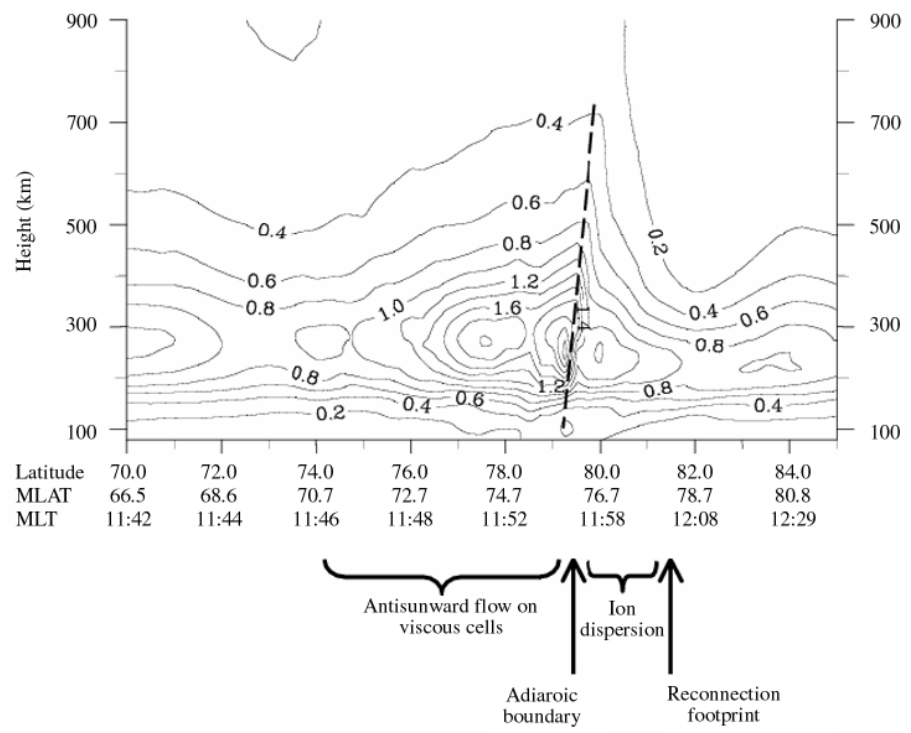

Fig. 16. Tomographic image showing signatures in the high-latitude ionosphere of reconnection at the magnetospheric lobe. From Pryse et al. (1999), reproduced by permission of the American Geophysical Union.

then carried zonally by viscous processes. It should be noted that the trajectory of the satellite, which was to the east of the chain of observing stations, gives rise to a geometrical distortion resulting in the apparent reverse slope with altitude of the field-aligned boundary.

Tomographic images from observations at high latitudes have also been used to investigate other signatures of the impact of space-weather processes in the ionospheric plasma. For example, Moen et al. (1998) related structures in electron density seen in tomographic images to optical aurorae, with the precipitation responsible for green-line emissions being linked to density enhancements in the $E$-layer, while the softer particles given rise to red-line aurora produced discrete features in the plasma at $F$-layer heights.

\section{Conclusions}

The results reviewed here demonstrate the potential role of radio tomographic imaging of the ionized atmosphere in both applied radio science, directed towards the mitigation of propagation effects on practical radio systems, and in studies of the geophysics of the underlying physical processes. The experimental technique is able to provide a unique capability to monitor large-scale spatial structures in the electron density over a wide region from observations made at a limited number of ground stations. The advantages of method, given by wide coverage even in inaccessible and environmentally hostile regions, coupled with the low cost of a passive system, far out way the known limitations. The latter result primarily from the lack of horizontal ray paths in satellite-to-ground propagation, though they can be mitigated by use of appropriate reconstruction algorithms and with additional input from ionosondes or other independent sources. The recent studies outlined here have shown the potential of radio tomography in fields as diverse as the validation of both empirical and physical models of the ionosphere, oblique sounding, $H F$ ray tracing, direction finding and the mapping of ionospheric parameters. The versatility of tomographic imaging in applied radio science adds to the potential of the method as a new experimental technique that can be used in geophysical studies to identify signatures of space-weather processes in the ionized 
atmosphere at high latitudes. A fuller review of the wider use of tomographic imaging in global aspects of geophysics can be found in Pryse (2003).

\section{Acknowledgements}

The development of radio tomography by the Radio and Space Physics Group at UWA has been supported financially at various stages by the U.K. Particle Physics and Astronomy Research Council, the Radiocommunications Agency, DERA and EOARD. The important contributions of many individuals, both within the group and as external scientific collaborators are acknowledged with grateful thanks, as is the assistance of many people and organisations for the practical support provided for the experimental activities.

\section{REFERENCES}

Balthazor, R.L., M.H. Denton, L. Kersley, S.E. Pryse, I.K. WALKer, R.J. MofFETT and G.J. BAILEY (2002): The High-latitude Dayside trough under Quiet Geomagnetic Conditions: a Comparison of Modelled and Experimental Observations (unpublished manuscript, University of Sheffield, U.K.).

Bilitza, D., K. Rawer, L. Bossy and T. Gulyaeva (1993): International reference ionosphere - Past, present and future: 1 Electron density, Adv. Space Res., 13, 3-13

BRADLEY, P.A. (1999): Prediction and Retrospective Ionospheric Modelling over Europe (PRIME), in COST Action 238 Final Report, published by Rutherford Appleton Laboratory, Chilton, Didcot, U.K..

DABAS, R.S. and L. KeRSLEY (2003): Radio tomographic imaging as an aid to modelling of ionospheric electron density, Radio Sci., 38 (3), 1-20.

DANIELl, R.E. JR., L.D. Brown, D.N. ANDERSON, M. W. Fox, P.H. DoherTy, D.T. DeCKer, J.J. SoJKa and R.W. SCHUNK (1995): PIM: a global ionospheric parameterization based on first principles, Radio Sci., 30, 1499-1510.

Fremouw, E.J., J.A. SeCAN and B.M. Howe (1993): Application of stochastic inversion theory to ionospheric tomography, Radio Sci., 17, 721-732.

Heaton, J.A.T., P.S. CANnON, N.C. Rogers, C.N. MitChell and L. KeRSLEY (2001): Validation of electron density profiles derived from oblique ionograms over the United Kingdom, Radio Sci., 36, 1149-1156.
Idenden, D.W., R.J. Moffett, M.J. Williams, P.J.S. SPENCER and L. KERSLEY (1998): Imaging of structures in the high-latitude ionosphere: model comparisons, Ann. Geophysicae, 16, 969-973.

KaK, A.C. and M. Slaney (1988): Principles of Computerised Tomographic Imaging (IEEE Press), pp. 327.

Kersley, L., J.A.T. Heaton, S.E. Pryse and T.D. Raymund (1993): Experimental ionospheric tomography with ionosonde input and EISCAT verification, Ann. Geophysicae, 11, 1064-1074.

Kersley, L., S.E. Pryse, I.K. Walker, J.A.T. Heaton, C.N. MitChEll, M.J. Williams and C.A. Willson (1997): Imaging of electron density troughs by tomographic techniques, Radio Sci., 32, 1607-1621.

Kersley, L., D. Malan, S.E. Pryse, Lu.R. Cander and R. BAMFORD (2002): Progress towards verification and validation of TEC observations over Europe, in Proceedings of the COST271 Workshop, September 2002, Faro, Portugal.

Kunitsyn, V.E. and E.D. TereshChEnKo (2003): Ionospheric Tomography (Springer-Verlag), pp. 260.

LEITINGER, R. (1996): Tomography, in Modern Ionospheric Science - 50 Years of Ionospheric Research in Lindau, edited by H. KoHL, R. RÜsteR and K. SCHLEgEL, 346370

LeITINGER, R. (1999): Ionospheric Tomography, in Review of Radio Science, 1996-1999, edited by W.R. STONE (Oxford University Press, Oxford, U.K.).

LocKWOOD, M. (1998): Identifying the open-closed field line boundary, in Polar Cap Boundary Phenomena, edited by J. Moen, A. Egeland and M. Lockwood (NATO Advanced Study Institute Series, Kluwer Academic Press, The Netherlands), 509, 73-90.

Moen, J., S.T. Berry, L. Kersley and B. LybeKK (1998): Probing discrete auroral arcs by ionospheric tomography, Ann. Geophysicae, 16, 574-582.

PRYSE, S.E. (2003): Radio tomography: a new experimental technique, Surv. Geophys., 24, 1-38.

Pryse, S.E., A.M. Smith, J. Moen and D.A. LoRentZen (1999): Footprints of lobe reconnection observed in ionospheric electron density under steady northward IMF, Geophys. Res. Lett., 26, 25-28.

Rogers, N.C., C.N. Mitchell, J.A.T. Heaton, P.S. CANNON and L. KERSLEY (2001): The application of radio tomographic imaging to $H F$ oblique incidence ray tracing, Radio Sci., 36, 1591-1598.

Walker, I.K., J. Moen, C.N. Mitchell, L. Kersley and P.E. SANDHOLT (1998): Ionospheric effects of magnetopause reconnection observed using ionospheric tomography, Geophys. Res. Lett., 25, 293-296.

Warrington, E.M., A.J. Stocker, N.Y. ZaAlov, D.R. SiDDLE and T.B. JONES (2002): High latitude ionospheric propagation effects and their influence on $H F$ direction finding, in Proceedings of the Fourth Symposium on 'Radiolocation and Direction Finding', May 2002, Southwest Research Institute, San Antonio, Texas, U.S.A. 